\title{
Right ventricular involution: What can we learn from nature's model of compensated hypertrophy?
}

\author{
Megan E. Bowen, MD, ${ }^{a}$ Xiaoqing Liu, MD, ${ }^{\text {a }}$ Peter M. Sundwall, BS, ${ }^{\text {a }}$ Stavros G. Drakos, MD, PhD, ${ }^{b}$ \\ Dean Y. Li, MD, PhD, ${ }^{b}$ Craig H. Selzman, MD, and Stephen H. McKellar, MD, MSc ${ }^{a}$
}

\begin{abstract}
Background: Right ventricular (RV) failure (RVF) is a vexing problem facing patients with various disease processes and carries a high mortality. RVF is a poorly understood phenomenon with limited treatment options. In mammalian fetal circulation, the right ventricle is the systemic ventricle. In neonates, however, the left ventricle assumes that role and gradually thickens compared with the right ventricle. This process, known as right ventricular involution (RVI), is poorly understood. We sought to define the time course and identify mechanisms involved in RVI.
\end{abstract}

Methods: Wild-type mice were bred and sacrificed on day of life (DOL) 1, 4, 8, 16 , and 30 to evaluate left ventricular (LV) and RV wall thickness and apoptosis. A terminal deoxynucleotidyl transferase nick-end labeling assay and RNA sequencing were performed to measure changes during RVI.

Results: Morphometric analysis demonstrated the changes in RV and LV wall thickness occurring between DOL 1 and DOL 16 (RV:LV, 0.53:0.44; $P=.03$ ). In addition, apoptosis was most active early, with the highest percentage of apoptotic cells on DOL $1(1.0 \%)$ and a significant decrease by DOL 30 $(0.23 \%)(P=.02)$. Similarly, expression of the proapoptotic genes BCL2l11 and Pawr were increased at DOL 1, and the antiapoptotic genes Nol3 and Naip2 were significantly increased at DOL 30.

Conclusions: RVI is a misnomer, but significant changes occur early (by DOL 16) in neonatal mouse hearts. Apoptosis plays a role in RVI, but whether manipulation of apoptotic pathways can prevent or reverse RVI is unknown and warrants further investigation. (J Thorac Cardiovasc Surg 2018;155:2024-8)

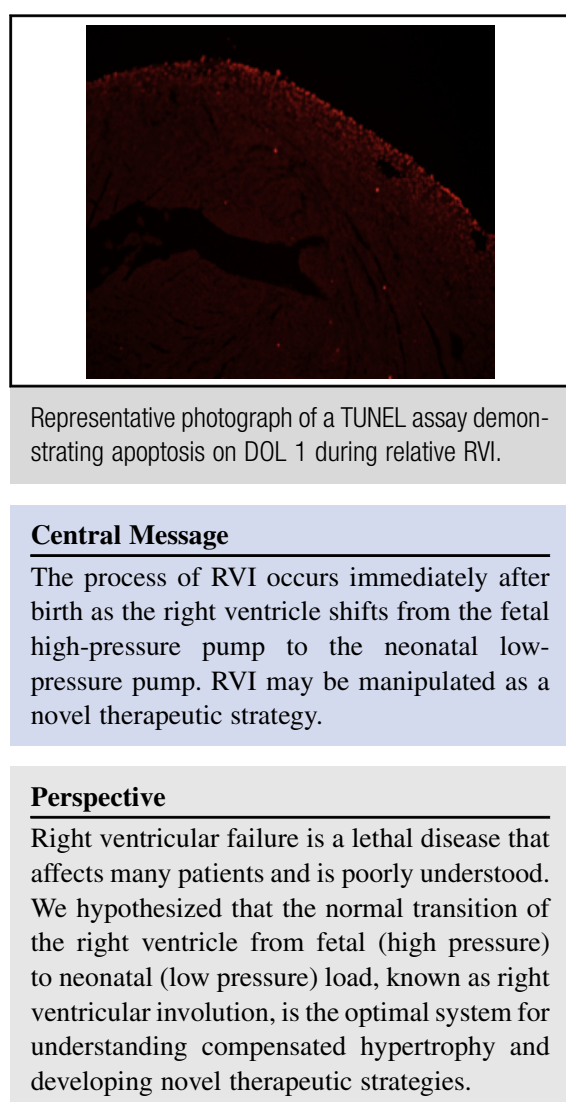

See Editorial Commentary page 2029.
From the ${ }^{\mathrm{a}}$ Division of Cardiothoracic Surgery, Department of Surgery, and ${ }^{\mathrm{b}}$ Department of Molecular Medicine, University of Utah School of Medicine, Salt Lake City, Utah.

This research was supported by the National Institutes of Health (Ruth L. Kirschstein National Research Service Award 5T32HL007576-30 and Grant R01 HL089592 from the National Heart, Lung, and Blood Institute), an American College of Surgeons Faculty Research Scholarship, and an American Association for Thoracic Surgery Grahm Foundation Award.

Received for publication May 11, 2017; revisions received Nov 24, 2017; accepted for publication Dec 6, 2017; available ahead of print Jan 19, 2018.

Address for reprints: Stephen H. McKellar, MD, MSc, Division of Cardiothoracic Surgery, University of Utah, 3C127 SOM, Salt Lake City, UT 84132 (E-mail: stephen.mckellar@hsc.utah.edu).

0022-5223/\$36.00

Copyright (c) 2017 by The American Association for Thoracic Surgery

https://doi.org/10.1016/j.jtcvs.2017.12.042
Right ventricular (RV) failure (RVF) is an important clinical problem associated with high mortality. Several patient populations suffer from RVF, including patients with congenital heart disease, ${ }^{1}$ pulmonary arterial hypertension $(\mathrm{PAH}),{ }^{2}$ and ischemic and nonischemic cardiomyopathies. ${ }^{3}$ Whereas left ventricular (LV) failure is well understood and

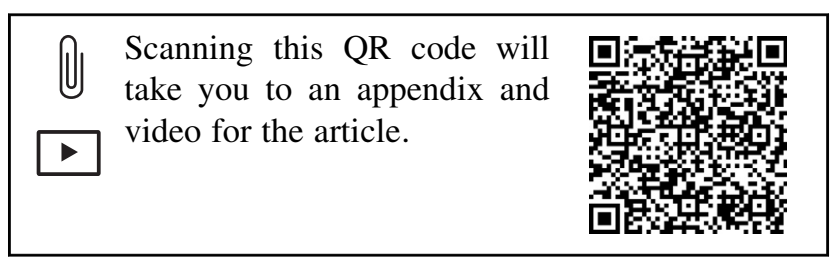




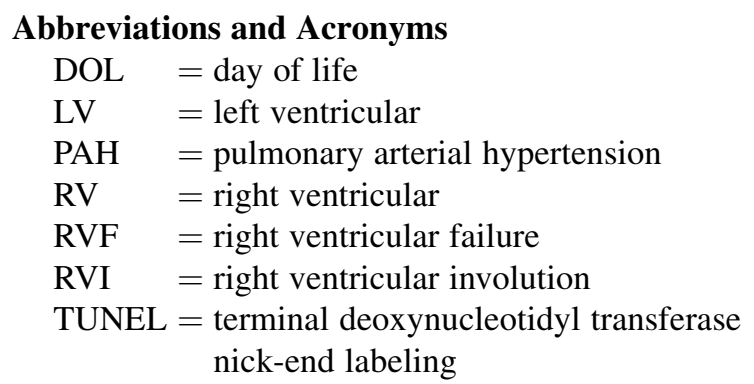

has good therapeutic options, RVF is poorly understood, with only limited replacement options, including use of a temporary extracorporeal blood pump, non-Food and Drug Administration-approved durable biventricular assist devices, and a total artificial heart. There is an important need to better understand and treat RVF.

$\mathrm{RV}$ involution (RVI) is the process through which the thickened neonatal right ventricle thins and remodels into its adult morphology. This change is a normal response to the decreased RV afterload that occurs during the shift from fetal circulation to adult circulation. In the fetal circulation, two-thirds of the cardiac output goes through the right ventricle and through the ductus arteriosus, making it the systemic ventricle. ${ }^{4}$

Despite being poorly understood, the right ventricle has clinical significance, observed most strikingly in Eisenmenger's syndrome. ${ }^{1}$ Researchers have reported better patient survival among patients with Eisenmenger's syndrome compared with other forms of pulmonary hypertension, and have postulated that this is due to the lack of RVI at birth, resulting in a neonatal phenotype. ${ }^{5}$ Patients with Eisenmenger's syndrome, in contrast to those with other forms of PAH, tend to die as a result of pulmonary processes rather than of RVF. This observation demonstrates the difference between patients who do and do not experience RVI before increased RV afterload.

Very little is known about the structural and molecular features of RVI, however. What is known is limited to the fate of the cardiac conduction system. ${ }^{6}$ Whether understanding the biology of RVI and potentially manipulating the heart to recreate the neonatal phenotype has not yet been investigated (Video 1).

We hypothesize that the cellular and molecular environment of the neonatal mammalian right ventricle before RVI is the natural model of compensated, sustainable, RV hypertrophy. We further hypothesize that understanding that neonatal milieu to recreate it may yield therapeutic insight into how to prevent RVF and promote right ventricle recovery in humans. Thus, we sought to define the time course of RVI in neonatal mouse hearts and explore the transcriptome and biological processes

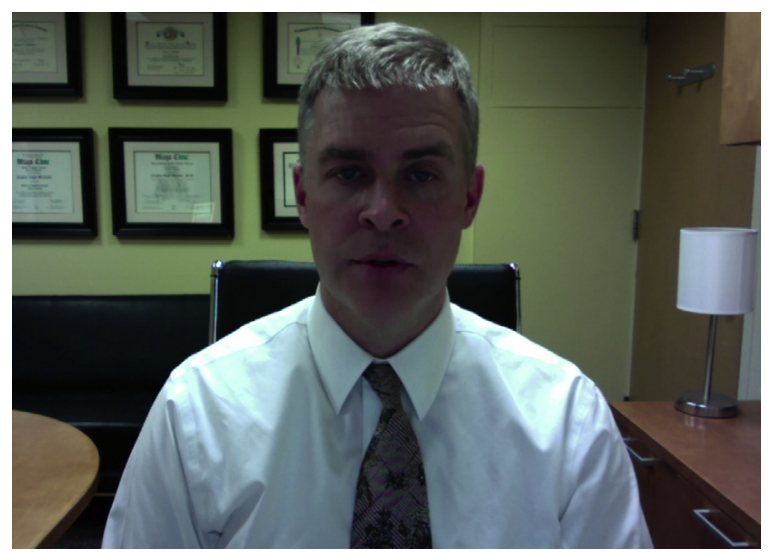

VIDEO 1. One of the authors contextualizes the rationale for studying neonatal mouse hearts to better understand right ventricular failure and recovery. Video available at: http://www.jtcvsonline.org/article/S00225223(17)32888-X/fulltext.

involved in RVI, focusing on the roles of apoptosis and autophagy in the process of RVI.

\section{METHODS}

The study protocol was reviewed and approved by the University of Utah's Institutional Animal Care and Use Committee. Twenty-seven C57BK/6NCrl wild-type mice (Charles River Laboratories, Wilmington, Mass) were bred and assigned to be sacrificed on day of life (DOL) 1 $(\mathrm{n}=6), 4(\mathrm{n}=5), 8(\mathrm{n}=5), 16(\mathrm{n}=6)$, or $30(\mathrm{n}=5)$, to evaluate the time course of RVI from the neonatal right ventricle through near-adulthood. Hearts were removed, sectioned, and immediately snap-frozen for RNA analysis or maintained in $10 \%$ buffered formalin (Thermo Fisher Scientific, Waltham, Mass) for 48 hours for morphometric analysis. For histological analysis, after fixation, the tissues were stained with periodic acid Schiff (Sigma-Aldrich, St Louis, Mo) as described previously. ${ }^{7}$ Digital images were captured from PAS-stained slides, and whole-field digital microscopy was performed with an Aperio ScanScope XT (Leica Microsystems, Buffalo Grove, Ill). ImageJ (National Institutes of Health, Bethesda, Md) was used to measure ventricular wall thickness.

Apoptosis was measured using 3 samples each from DOL 1, 4, 8, and 30. The In Situ Cell Death Detection Kit, TMR red (Roche Applied Science, Indianapolis, Ind) was used to label apoptosis cell DNA strand breaks and detect them under fluorescence microscopy. Slides were visualized with CellSens DP72 (Olympus America, Hackensack, NJ) by fluorescence microscopy, obtaining consecutive $20 \times$ magnification images of a single field with fluorescein isothiocyanate and DAPI filters. The apoptotic index (AI) was calculated as $\mathrm{AI}=$ (number of terminal deoxynucleotidyl transferase nick-end labeling [TUNEL]-positive cells/total number of cells) $\times 100$.

For gene expression analysis, RNA was extracted from RV tissue from all 27 mice using TRIzol and homogenized using TissueLyser II (Qiagen, Newton, Pa) and a PureLink RNA Mini Kit (Life Technologies, Carlsbad, Calif). RNA concentration was measured with a Nanodrop 2000 spectrophotometer (Thermo Fisher Scientific).

Illumina TruSeq Stranded Total RNA Sample Prep with Ribo-Zero Human/Mouse/Rat Library construction was performed using the Illumina TruSeq Stranded Total RNA Sample Preparation Kit with Ribo-Zero Human/Mouse/Rat (catalog nos. RS-122-2201 and RS-122-2202; Illumina, San Diego, Calif).

RNA sequencing was performed using an Illumina HiSeq 50 cycle single-read sequencing version 4 . Sequencing libraries (25 pM) were chemically denatured were applied to an Illumina HiSeq v4 single-read 
TABLE 1. Morphological analysis and apoptosis

\begin{tabular}{lcccccccc}
\hline \multicolumn{1}{c}{ Variable } & Number per group & DOL1 & DOL4 & DOL8 & DOL16 & DOL30 & P value, DOL1 vs DOL30 \\
\hline RV thickness, $\mu \mathrm{M}$ & $5-6$ & $352 \pm 48$ & $311 \pm 86$ & $417 \pm 108$ & $607 \pm 54$ & $667 \pm 73$ & $<.001$ \\
LV thickness, $\mu \mathrm{M}$ & $5-6$ & $668 \pm 54$ & $676 \pm 97$ & $791 \pm 105$ & $1324 \pm 237$ & $1529 \pm 58$ & $<.001$ \\
RV:LV & $5-6$ & $0.53 \pm 0.08$ & $0.46 \pm 0.1$ & $0.52 \pm 0.1$ & $0.47 \pm 0.08$ & $0.44 \pm 0.04$ & .03 \\
Apoptosis, \% & 3 & 1 & 0.39 & 0.32 & NA & 0.24 & .02 \\
\hline
\end{tabular}

$D O L$, Day of life; $R V$, right ventricular; $L V$, left ventricular; $N A$, not available.

flow cell using an Illumina cBot. Hybridized molecules were clonally amplified and annealed to sequencing primers with reagents from an Illumina HiSeq SR Cluster Kit v4-cBot (GD-401-4001). Following transfer of the flow cell to an Illumina HiSeq instrument (HCSv2.2.38 and RTA v1.18.61), a 50-cycle single-read sequence run was performed using HiSeq SBS Kit v4 sequencing reagents (FC-401-4002).

\section{Analysis of RV Transcripts}

Reads were aligned to the genome (build $\mathrm{mm} 10$ ) with included splice junction sequences from all known transcripts (Ensembl annotation) using Novoalign version 2.8 (Novocraft, Selangor, Malaysia), allowing for up to 50 matches. Alignments to splice junctions were converted to genomic coordinates using the USeq application SamTranscriptomeParser (USeq version 8.8.1; https://github.com/HuntsmanCancerInstitute/USeq), allowing for a maximum of 1 random placement for multiple-mapping alignments. Normalized depth coverage (per million mapped reads) tracks were generated using USeq applications Sam2USeq and USeq2UCSC. Differential gene expression was determined using the USeq application DefinedRegionDifferentialSeq, which is a wrapper around DESeq2 (http:// www.bioconductor.org/packages/release/bioc/html/DESeq2.html), using default parameters and Ensembl annotation with collapsed transcripts. Differentially expressed genes were identified using a threshold of absolute $\log 2$ fold change $>1$ and a $-10 \log 10$-adjusted $P$ value $>13$ (or approximately $5 \%$ false-discovery rate). Pathways were generated using Ingenuity Pathway Analysis (Qiagen). Violin plots were generated using custom R scripts (analysis/Plots). Alignment metrics were generated using Picard CollectRnaSeqMetrics (http://broadinstitute.github.io/picard/index.html).

Continuous data are presented as mean $\pm \mathrm{SD}$ where appropriate, and nonparametric analyses were performed due to the assumption of a non-normal distribution based on sample size. The Kruskal-Wallis test was used for continuous variables with multiple comparisons, as appropriate (Table 1). Statistical analysis for nonparametric comparisons was performed using JMP (SAS Institute, Cary, NC) and Prism 7 (GraphPad Software, La Jolla, Calif). The cutoff for statistical significance was $P<.05$.

\section{RESULTS}

Our morphometric analysis revealed the time course of RVI with respect to RV wall thickness, both by itself and compared with the left ventricle (Figure 1). Overall, RV wall thickness increased from $352 \pm 48 \mu \mathrm{m}$ on DOL 1 to $667 \pm 73 \mu \mathrm{m}$ on DOL $30(P<.001)$. Similarly, LV thickness increased from $668 \pm 54 \mu \mathrm{m}$ to $1529 \pm 58 \mu \mathrm{m}(P<.001)$ (Table 1$)$. The relative RV thickness compared with LV thickness decreased over time, however, from a ratio of 0.53 on DOL 1 to 0.44 on DOL $30(P=.03)$. Looking at the ventricular wall thicknesses across the entire time spectrum, it appears that most of the changes in RV and LV thickness occur by DOL 16, making DOL 8 the last time point sampled with relevant RVI biology for the remainder of the analyses. Accordingly, we performed the apoptosis and autophagy analyses on DOL 1,8 , and 30 .

We performed a TUNEL assay to evaluate the degree of apoptosis involved in RVI and observed a significant
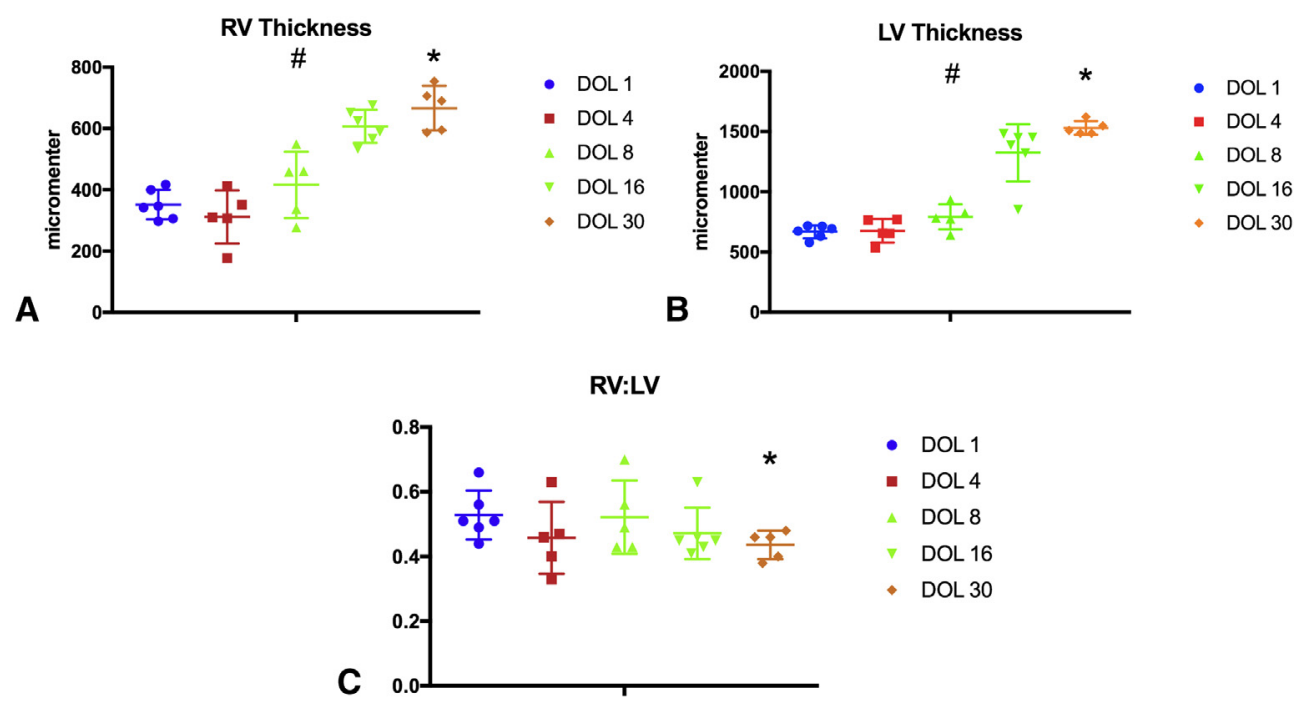

FIGURE 1. Right ventricular $(R V)(\mathrm{A})$ and left ventricular $(L V)(\mathrm{B})$ wall thickness over time in neonatal mouse hearts demonstrating increasing thickness of the ventricular free wall in both the right and left ventricles and decreasing RV:LV ratio. C, Thickness in RV involution. \#P $<.01$ for all groups, Kruskal-Wallis test; ${ }^{*} P<.01$ versus day of life 1 , post hoc analysis. $D O L$, Day of life. 


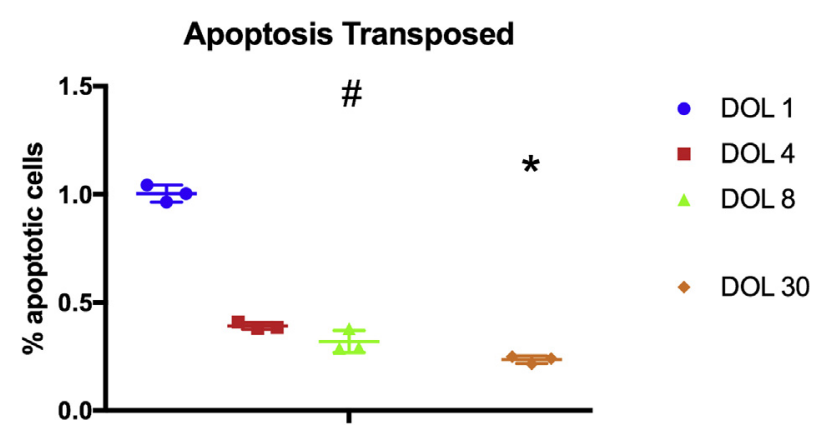

FIGURE 2. Terminal deoxynucleotidyl transferase nick-end labeling assay demonstrating the decreasing percentage of apoptotic cells detected in right ventricular tissue over time. $\# P<.01$ for all groups, Kruskal-Wallis test; ${ }^{*} P<.01$ versus day of life 1 , post hoc analysis. $D O L$, Day of life.

decrease over time. The percentage of apoptotic cells dropped from $1.0 \%$ on DOL 1 to $0.23 \%$ on DOL 30 $(P=.02)$ (Figure 2 and Table 1$)$. We found support for this observation at the transcriptome level of apoptosis-related genes. On DOL 1, there was increased expression of the proapoptotic genes Bcl2ll1, Pawr, and Aifm2. Similarly, on DOL 30, there was decreased expression of the antiapoptotic genes Nol3, Naip2, Naip5, and Naip6 (Table 2).

We explored a possible role for authophagy in RVI and indeed found a mixed signal at the transcriptome level. We observed increased expression of Deptor, Pik3rl,

TABLE 2. Gene Expression at DOL 30 versus DOL 1

\begin{tabular}{|c|c|c|}
\hline Gene & $\log 2$ & $P$ value \\
\hline \multicolumn{3}{|l|}{ Antiapoptotic } \\
\hline Nol3 & 1.9 & $<.01$ \\
\hline Naip5 & 1.6 & $<.01$ \\
\hline Naip2 & 1.6 & $<.01$ \\
\hline Naip6 & 1.4 & $<.01$ \\
\hline \multicolumn{3}{|l|}{ Proapoptotic } \\
\hline Aifm3 & -1.7 & .02 \\
\hline Pawr & -1.4 & $<.01$ \\
\hline Bcl2l11 & -1.3 & $<.01$ \\
\hline \multicolumn{3}{|l|}{ Autophagy } \\
\hline Deptor & 1.8 & $<.01$ \\
\hline Pik3rl & 1.4 & $<.01$ \\
\hline $\operatorname{Atg} 9 a$ & 1.1 & $<.01$ \\
\hline Becn1 & 0.01 & .90 \\
\hline Mtor & 0.15 & .08 \\
\hline Map1lc $3 b$ & 0.86 & $<.01$ \\
\hline Lamp1 & 0.46 & .02 \\
\hline \multicolumn{3}{|c|}{ Glucose metabolism } \\
\hline Glut4 & 2.2 & $<.01$ \\
\hline$P d k 2$ & 1.8 & $<.01$ \\
\hline$P d k 4$ & 1.1 & $<.01$ \\
\hline$P d k 3$ & -2.6 & $<.01$ \\
\hline \multicolumn{3}{|c|}{ Contractile proteins } \\
\hline Actal & 1.7 & $<.01$ \\
\hline Myh10 & -1.7 & $<.01$ \\
\hline
\end{tabular}

Atg9a, Mapllc3b, and Lampl at DOL 30 compared with DOL 1, but no difference in the common autophagy genes Becnl and Mtor (Table 2).

When taking a broader view of the transcriptome in the process of RVI, such as fuel metabolism and contractile proteins, we observed differential expression of genes involved in fuel metabolism. Specifically, we observed increased expression of genes involved in glucose uptake (Glut4) and the pyruvate dehydrogenase (PDH) inhibitors Pdk2 and Pdk4 at DOL 30 compared with DOL 1, suggesting better glucose metabolism as the right ventricle matures. In addition, we observed an expected decrease in the contractile protein Myh10 encoding beta-myosin heavy chain $(\beta$-MHC) at DOL 30.

\section{DISCUSSION}

The principal finding of the present study is that the process of RVI is a misnomer, but changes do occur starting immediately after birth and are nearly completed by DOL 16. These data show that during RVI, RV thickness increases overall, but decreases relative to LV thickness. We also observed that apoptosis, but not autophagy, plays a role in RVI.

The term RVI appears to be a misnomer, given that absolute RV thickness actually increases throughout the neonatal period, but decreases in relation to LV thickness. The relative decrease in RV thickness is an anticipated and normal physiological response to the sudden decrease in RV afterload in neonatal mammals. Perhaps a more precise term, such as relative RVI, would be more appropriate. The time course of this phenomenon is now known (at least in mice) and, surprisingly, appears to be rapid and nearly completed by DOL 16 . The implications of the rapid time course of relative RVI is consistent with what we observe clinically in patients with Eisenmenger's syndromes, whose cardiac lesions exist from birth and preclude RVI. But even now, when we know the time course of relative RVI, many unknowns remain-specifically, which biological processes remain active in Eisenmenger hearts compared with those that undergo RVI? Another consideration is whether this process of RVI involves similar biological processes observed in studies of LV unloading with $\mathrm{LV}$ assist devices in humans. ${ }^{8}$

\section{Apoptosis}

We observed that the process of apoptosis was more active in the early stages of RVI and decreased gradually over time. The percentage of apoptotic cells dropped gradually from a peak of $1 \%$ on DOL 1 to $0.23 \%$ by DOL 30. Our analysis of the transcriptome supports our TUNEL findings of more active apoptosis at DOL 1. Compared with DOL 1, we observed increased expression of the antiapoptotic genes Nol3, Naip2, Naip5, and Naip6, and decreased expression of proapoptotic genes Bcl2ll1, 
Pawr, and Aifm3 at DOL 30. Thus, apoptosis may be a potential therapeutic target to maintain the milieu of neonatal relative RVI. Apoptosis inhibitors are currently being evaluated as research tools to help explain the cellular mechanisms underlying stroke, spinal cord injuries, multiple sclerosis, atherosclerosis, heart disease, cancer, and other neurodegenerative diseases. ${ }^{9}$ These compounds are inhibitors of the Bax, c-Myc, p-53, tBid, Bcl, caspase, and other pathways that mediate apoptosis, but whether any of these agents may play a role in preventing RVI remains to be studied.

\begin{abstract}
Autophagy
Although autophagy was included in our original hypothesis, our transcriptomic observations were somewhat mixed. We observed increased expression in autophagy genes Deptor, Pik3rl, Atg9a, Mapllc3b, and Lampl at DOL 30 compared with DOL 1. Regardless of the mixed picture, the lack of differential gene expression in the early neonatal period suggests that autophagy is not active before RVI and thus is not a likely therapeutic target for maintaining or recreating the pre-RVI milieu, but only electron microscopic analysis would be conclusive.
\end{abstract}

\section{Other Pathways}

When taking a broader look at the transcriptome for clues to the processes involved in relative RVI, we observed differential gene expression in contractile proteins as well as fuel metabolism. As in humans, ${ }^{10}$ we observed decreased expression of Myh10 which encodes for $\beta$-MHC later in life, suggesting that more $\beta$-MHC may be a compensatory component in the neonatal right ventricle, rather than a deleterious sign of a failing heart. Because $\beta$-MHC is also expressed in humans with heart failure, ${ }^{11}$ identifying therapies to manipulate the ratios of myosin isoforms may be a therapeutic strategy meriting further study.

Similarly, we observed changes in gene expression for genes involved in fuel metabolism. Expression of Glut4, which facilitates increased glucose uptake, and 2 isoforms of PDK, was increased at DOL 30 compared with DOL 1, suggesting a different mix of energy substrate later in life, but the implications of these observations are unclear without static or flux metabolomic data. Further research on manipulating fuel metabolism to recreate the metabolic profile of the neonatal, compensated right ventricle is warranted.

Now that the time course of relative RVI has been established on a morphologic level, we believe that studying this physiological model of compensated RV hypertrophy could be highly productive, not only to better understand this fascinating biological phenomenon, but also to recreate the neonatal milieu in adult hearts to treat heart failure. This concept is not new; reverting from an adult program to a neonatal program during heart failure is well established for contractile proteins, such as MHC. ${ }^{10,12}$ Nonetheless, further research is needed to elucidate which parts of the neonatal milieu are favorable and which are pathological, as well as which can be manipulated therapeutically. Animal models exist for creating high PAH (such as monocrotaline) that could be given to neonatal mice in an attempt to preserve this physiological model of compensated hypertrophy. ${ }^{13}$

\section{CONCLUSIONS}

Relative RVI occurs rapidly in neonatal mouse hearts and is associated with changes in apoptosis and differential gene expression in contractile proteins and fuel metabolism. These potential therapeutic targets warrant further investigation in an attempt to recreate the milieu of compensated RV hypertrophy found in neonatal mammals, which could yield therapies for right ventricle protection or recovery in humans with RVF.

\section{Conflict of Interest Statement}

Authors have nothing to disclose with regard to commercial support.

\section{References}

1. Hopkins WE, Waggoner AD. Severe pulmonary hypertension without right ventricular failure: the unique hearts of patients with Eisenmenger syndrome. Am J Cardiol. 2002;89:34-8.

2. McLaughlin VV, Presberg KW, Doyle RL, Abman SH, McCrory DC, Fortin T, et al. Prognosis of pulmonary arterial hypertension: ACCP evidence-based clinical practice guidelines. Chest. 2004;126(1 Suppl):78S-92S.

3. Drakos SG, Janicki L, Horne BD, Kfoury AG, Reid BB, Clayson S, et al. Risk factors predictive of right ventricular failure after left ventricular assist device implantation. Am J Cardiol. 2010;105:1030-5.

4. Fineman JR, Soifer SJ. The fetal and neonatal circulations. In: Chang AC, Hanley F, Wernovsky G, Wessel DL, eds. Pediatric Cardiac Intensive Care. Baltimore, MD: Lippincott Williams \& Wilkins; 1998:17-22.

5. Hopkins WE. The remarkable right ventricle of patients with Eisenmenger syndrome. Coron Artery Dis. 2005;16:19-25.

6. Ottaviani G, Matturri L, Rossi L, James TN. Crib death: further support for the concept of fatal cardiac electrical instability as the final common pathway. Int J Cardiol. 2003;92:17-26.

7. McKellar SH, Javan H, Bowen ME, Liu X, Schaaf CL, Briggs CM, et al. Animal model of reversible, right ventricular failure. J Surg Res. 2015;194:327-33.

8. Diakos NA, Selzman CH, Sachse FB, Stehlik J, Kfoury AG, Wever-Pinzon O, et al. Myocardial atrophy and chronic mechanical unloading of the failing human heart: implications for cardiac assist device-induced myocardial recovery. J Am Coll Cardiol. 2014;64:1602-12.

9. Sureda FX, Junyent F, Verdaguer E, Auladell C, Pelegri C, Vilaplana J, et al. Antiapoptotic drugs: a therapautic strategy for the prevention of neurodegenerative diseases. Curr Pharm Des. 2011;17:230-45.

10. Razeghi P, Young ME, Alcorn JL, Moravec CS, Frazier OH, Taegtmeyer H. Metabolic gene expression in fetal and failing human heart. Circulation. 2001; 104:2923-31.

11. Lowes BD, Zolty R, Shakar SF, Brieke A, Gray N, Reed M, et al. Assist devices fail to reverse patterns of fetal gene expression despite beta-blockers. $J$ Heart Lung Transplant. 2007;26:1170-6.

12. Rajabi M, Kassiotis C, Razeghi P, Taegtmeyer H. Return to the fetal gene program protects the stressed heart: a strong hypothesis. Heart Fail Rev. 2007; 12:331-43.

13. Piao L, Marsboom G, Archer SL. Mitochondrial metabolic adaptation in right ventricular hypertrophy and failure. J Mol Med (Berl). 2010;88:1011-20.

Key Words: right ventricle, involution, right heart failure 


\section{APPENDIX E1}

\section{Library Creation}

In brief, ribosomal RNA is removed from total RNA samples (100 ng to $4 \mathrm{ug}$ ) using biotinylated Ribo-Zero oligos attached to magnetic beads that are complimentary to cytoplasmic rRNA. Following purification, the rRNA-depleted sample is fragmented with divalent cations under elevated temperatures and primed with random hexamers in preparation for cDNA synthesis. First strand reverse transcription is accomplished using Superscript II Reverse Transcriptase (Invitrogen cat\#18064-014). Second strand cDNA synthesis is accomplished using DNA polymerase I and Rnase $\mathrm{H}$ under conditions in which dUTP is substituted for dTTP, yielding blunt-ended cDNA fragments in which the second strand with dUTP. An A-base is added to the blunt ends as a means to prepare the cDNA fragments for adapter ligation and block concatamer formation during the ligation step. Adapters containing a T-base overhang are ligated to the A-tailed DNA fragments. Ligated fragments are PCR-amplified
(12-15 cycles) under conditions in which the pcr reaction enables amplification of the first strand cDNA product, whereas attempted amplifciation of the second strand product stalls at dUTP bases and therefore is not represented in the amplified library. The PCR-amplified library is purified using Agencourt AMPure XP beads (Beckman Coulter Genomics cat\#A63881). Follwoing amplification, the library is purified by bead based methodologies. The concentration of the amplified library is measured with a NanoDrop spectrophotometer and an aliquot of the library is resolved on an Agilent 2200 Tape Station using a D1K (cat\# 5067-5361 and 5067-5362) or a High Sensitivity D1K (cat\# 5067-5363 and 5067-5364) assay to define the size distribution of the sequencing library. Libraries are adjusted to a concentration of approximately $10 \mathrm{nM}$ and quantitative PCR is performed using the KapaBiosystems Kapa Library Quant Kit (cat\# KK4824) to calculate the molarity of adapter ligated library molecules. The concentration is further adjusted following qPCR to prepare the library for Illumina sequence analysis. 